\title{
Assessing the Role of Muscle Protein Breakdown in Response to Nutrition and Exercise in Humans
}

\author{
Kevin D. Tipton $^{1}$ (D) D. Lee Hamilton ${ }^{1}$ Iain J. Gallagher ${ }^{1}$
}

Published online: 24 January 2018

(C) The Author(s) 2018. This article is an open access publication

\begin{abstract}
Muscle protein breakdown (MPB) is an important metabolic component of muscle remodeling, adaptation to training, and increasing muscle mass. Degradation of muscle proteins occurs via the integration of three main systems-autophagy and the calpain and ubiquitin-proteasome systems. These systems do not operate independently, and the regulation is complex. Complete degradation of a protein requires some combination of the systems. Determination of MPB in humans is technically challenging, leading to a relative dearth of information. Available information on the dynamic response of MPB primarily comes from stable isotopic methods with expression and activity measures providing complementary information. It seems clear that resistance exercise increases MPB, but not as much as the increase in muscle protein synthesis. Both hyperaminoacidemia and hyperinsulinemia inhibit the post-exercise response of MPB. Available data do not allow a comprehensive examination of the mechanisms behind these responses. Practical nutrition recommendations for interventions to suppress MPB following exercise are often made. However, it is likely that some degree of increased MPB following exercise is an important component for optimal remodeling. At this time, it is not possible to determine the impact of nutrition on any individual muscle protein. Thus, until we can develop and employ better methods to elucidate the role of MPB following exercise and the response to nutrition, recommendations to optimize post exercise
\end{abstract}

Kevin D. Tipton

k.d.tipton@stir.ac.uk

1 Physiology, Exercise and Nutrition Research Group, Faculty of Health Sciences and Sport, University of Stirling, Stirling, Scotland nutrition should focus on the response of muscle protein synthesis. The aim of this review is to provide a comprehensive examination of the state of knowledge, including methodological considerations, of the response of MPB to exercise and nutrition in humans.

\section{Introduction}

Skeletal muscle is a crucially important tissue for human health and well-being [1]. The importance of muscle for locomotion and strength is obvious. However, skeletal muscle also is the largest metabolically active tissue in the body. It is also the largest site for glucose disposal and acts as a fuel reserve for other organs during pathophysiological situations, including fasting. Thus, skeletal muscle is critical, not only for athletic performance, but for healthy daily living and aging. Understanding the regulation of gain or loss of muscle mass is therefore an important consideration for exercise and nutrition scientists.

Muscle proteins are constantly turning over, i.e., broken down (or degraded) and synthesized. The balance between the rates of synthesis and degradation of muscle protein pools, i.e., net muscle protein balance (NBAL), determines the amount of that protein in muscle. In particular, changes in the amount of muscle myofibrillar proteins lead to changes in muscle mass. Moreover, in addition to or instead of, modulating muscle mass, changes in muscle protein synthesis (MPS) and muscle protein breakdown (MPB) also may be crucial for repair and remodeling of muscle proteins following exercise [2]. So, the regulation of these processes is critical for optimal adaptation of muscle in terms of size. Thus, exercise and nutrition interventions that influence rates of MPS and MPB, and 
ultimately NBAL, have received increasing attention in the last two to three decades [2-5].

The influence of exercise and nutrition on the regulation of MPS is understood far better than MPB [2-5]. There are a number of reasons for this discrepancy. The study of MPB, particularly in humans, is technically much more difficult than MPS [6]. Furthermore, changes in MPS in response to exercise and nutrition have a much greater influence on NBAL than do changes in MPB [7, 8]. Also, the resolution of measuring MPS on a protein level is more readily accomplished than for MPB. Thus, the bulk of the studies attempting to contribute to our understanding of changes in muscle mass and in response to nutrition and exercise have focused on examining MPS [6]. Nevertheless, it is important to delineate the role of MPB for remodeling and repair of skeletal muscle in response to exercise and how nutrition influences these processes. This information contributes to our overall understanding of the metabolic processes behind muscle gains, losses, and repair and remodeling of muscle tissue leading to muscle adaptation. In this review, we will examine our current understanding of the process of MPB and how it responds to nutrition and exercise interventions and its role in changing skeletal muscle mass and adaptation. Thus, we will focus our discussion on data from studies in humans following exercise.

\section{Systems of Muscle Protein Breakdown}

There are three main systems that contribute to the catabolic component of muscle turnover; the ubiquitin-proteasomal pathway (UPP), autophagy, and the calpain $\mathrm{Ca}^{2+}$ dependent cysteine proteases. The best known of these processes is the UPP, which centers around the $26 \mathrm{kDa}$ proteasome that degrades proteins tagged with the $8.5 \mathrm{kDa}$ protein ubiquitin [9]. The UPP is central to protein degradation across all cell types and plays a fundamental role in normal physiology. E1 enzymes first activate ubiquitin. These enzymes capture ubiquitin and an ATP$\mathrm{Mg}^{2+}$ complex, and catalyse the acylation of the ubiquitin C-terminus and subsequent thioesterification, releasing adenosine monophosphate (AMP) in the process [10]. Ubiquitin is transferred to an E2 ubiquitin conjugating enzyme via transthioesterification [11]. Finally the activated ubiquitin is canonically transferred via an E3 ubiquitin ligase to a lysine group on a target protein [9]. The addition of four ubiquitin molecules to the target protein is the canonical signal for transfer of that protein to the $26 \mathrm{kDa}$ proteasome for degradation, but other non-canonical ubiquitination patterns have also been reported [12]. E3 ligases, e.g., muscle specific ring finger protein 1 (MuRF1) and atrogin1, have been the focus of much work after they were found to be elevated in several models of skeletal muscle atrophy $[13,14]$.

The UPP alone cannot degrade intact myofibrils $[15,16]$. Thus, there is a requirement for involvement of one or both of the other protein catabolic pathways, depending on the physiological situation. In terms of degrading sarcomeric proteins, it is believed that the calpain system (further described below) is required to break up sarcomeres into their component parts through the proteolytic activity of the calpains [17]. Much as the UPP and calpain systems are intricately linked to drive the destruction of specific proteins, so too is the autophagy pathway linked to the UPP [18]. Generally, the autophagy system involves the initial generation of an autophagosome surrounding bulk intracellular components or protein complexes. These components targeted for destruction could be intracellular organelles, damaged proteins or other target proteins (usually membrane bound proteins). The autophagosome then fuses with lysosomes leading to the degradation of the autophagosome contents. Several stressors activate autophagy in skeletal muscle, including reactive oxygen species generation and starvation.

The first step in the prototypical autophagy process is the formation of a nascent membrane structure, the phagophore. The origin of the membrane-whether endosomal, trans-Golgi, nuclear membrane or de novo synthesisis unclear. After the maturation of the autophagosome there is a fusion with lysosomes generating an autolysosome. Finally, activation of lysosomal proteases leads to the degradation of autolysosome contents and the recycling of amino acids. Thus, this system, in combination with the UPP, degrades proteins important for exercise performance and adaptation other than myofibrillar proteins, such as membrane bound proteins, e.g., transporters, ion channels, and receptors [19].

Calpains are non-lysosomal $\mathrm{Ca}^{2+}$-dependent cysteine proteases. Candidate targets for calpain activity in muscle include myofibrillar, cytoskeletal, and sarcolemmal proteins. There are three calpains expressed in skeletal muscle; calpain-1, calpain-2 (the ubiquitous calpains), and the muscle-specific calpain-3 [20]. Whereas calpain-1 and 2 require autolysation and heterodimerization with calpain-4, calpain-3 requires autolytic cleavage for activity but not dimerization to calpain-4. Once activated, calpain-1 and calpain- 2 are referred to as $\mu$ - or m-calpains, respectively, due to their reliance on micro- or millimolar concentrations of $\mathrm{Ca}^{2+}$ for activation. The requirement for millimolar $\mathrm{Ca}^{2+}$ levels for activation makes it difficult to discern a physiological role for m-calpain in skeletal muscle. Approximately $70 \%$ of $\mu$-calpain is thought to be freely available in the cytoplasm of skeletal muscle. Upon a rise in $\left[\mathrm{Ca}^{2+}\right]$, calpain- 1 dimerizes with calpain- 4 and binds to target proteins. Further sustained increases in $\left[\mathrm{Ca}^{2+}\right]$ are 
required for activation to $\mu$-calpain and the dissociation between target binding and activation is thought to be a mechanism to prevent inappropriate calpain driven proteolysis [21]. The ubiquitous calpains also are regulated by calpastatin. This regulation requires the heterodimeric form of the calpains and the presence of calcium [22]. Unlike calpain-1, calpain-3 is thought to be mostly bound to myofibrillar proteins, and in particular to titin [23]. The importance of calpain-3 in skeletal muscle homeostasis is underlined by the fact that lack of calpain-3 leads to limbgirdle muscular dystrophy type $2 \mathrm{~A}$ with sufferers becoming wheelchair-bound from early adulthood onwards [24].

It is clear that the three main protein degradation systems work simultaneously to contribute to the overall response of MPB in response to exercise and nutrition. Whereas mechanistic data are lacking from human studies, data from animal models suggest that the UPP and calpain systems play a much larger role than autophagy [17]. However, the autophagy system seems to be particularly important for degradation of receptor proteins at the membrane [19]. Given their importance in control of anabolic processes, control of receptor protein degradation plays an important role in muscle remodeling. Assessing markers of these pathways offers important mechanistic information leading to greater understanding of the role of MPB in muscle remodeling in response to exercise and nutrition.

\section{Methods for Measuring Muscle Protein Breakdown (MPB)}

Methods to assess the response of MPB to exercise and nutrition interventions in humans can be divided broadly into dynamic and static measurements. Measurements of the dynamic response of MPB are based primarily, albeit not entirely, on stable isotopic tracer methods. Static measurements stem primarily from assessing changes in molecular signals in muscle biopsy samples. All methods have their strengths and limitations. These considerations must be balanced with the level of invasiveness required for each method when choosing how best to assess the response of MPB. It is important to understand the strengths and limitations of methods used to measure MPB for optimal interpretation of the available data. We attempt to delineate these considerations for the methods discussed below.

\subsection{Dynamic Measures of MPB}

Stable isotopic tracer methods provide a powerful tool to determine metabolic responses to various perturbations, including nutrition and exercise. Arteriovenous (AV) blood sampling in combination with infusion of stable isotopically labeled amino acids has been used to assess MPS and MPB in vivo in humans [25]. This two-pool (arterial and venous amino acid pools) model allows calculation of the uptake and release of an amino acid that is not metabolized in muscle (such as phenylalanine) across the limb [26, 27]. The uptake and release are assumed to be due directly to MPS and MPB, respectively. For release of phenylalanine from the leg to represent MPB, it must be assumed that outward amino acid transport from the muscle into the venous pool is equivalent to MPB and both processes are in steady state $[26,27]$. So, MPB may be underestimated by the amount of amino acids that appear in the muscle intracellular pool that are reutilized for MPS and not transported out into the venous blood [28, 29]. Also, both physiological and isotopic steady-states are necessary for this model to offer robust results [26, 27, 30]. However, in many nutrition and exercise studies, physiological steady state is not possible. For example, when a bolus ingestion of amino acids or protein is a necessary component of the study design, transient expansion of the intracellular amino acid pool followed by amino acid efflux into the venous blood pool will result [31]. This transient expansion must be accounted for when calculating MPB. Thus, measurement of MPB in these situations is less reliable. Therefore, this two-pool model for estimation of MPB may be reliable and useful in certain situations, e.g., studies in which physiological and isotopic steady states are possible. However, the limitations must be considered carefully, particularly in studies involving bolus ingestion of a source of amino acids following exercise.

An important limitation of the two-pool AV balance model that must be considered is that it underestimates the true rate of MPB depending on the rate of amino acid transport, as well as the reutilization of intracellular amino acids for MPS. Thus, more recently an AV model was developed to determine the actual rate of appearance of amino acids into the intracellular pool from MPB [28, 29]. This three-pool (arterial, venous, and muscle intracellular) model provides a closer approximation of the true rate of MPB. In addition to the arterial and venous blood samples, the isotopic enrichment of intracellular amino acid tracers is determined from muscle biopsy samples. As with the two-pool model, both physiological and isotopic steady state are assumed with this three-pool model [28, 29]. Thus, the three-pool model is a refinement of the two-pool AV model, but limitations remain.

The AV balance models have been used to provide important information about muscle metabolism following exercise, including MPB. Yet, the limitations inherent with these models require careful consideration when interpreting results. The most commonly used limbs are the leg and forearm. Since samples are taken from venous blood 
draining an entire limb (the femoral vein drains the entire leg, not just the muscle tissue) calculation of MPB includes contributions from non-muscle tissues (e.g., skin, bone etc.). Biolo et al. [29] determined that muscle accounts for $85-90 \%$ of the metabolism of the leg at rest $[3,7]$. The contribution of non-muscle tissue is likely more for the forearm [3]. Given that exercise increases the metabolism of the muscle with little impact on other tissues, it is a reasonable assumption that measurements made using these AV models following exercise represent changes in muscle metabolism [3, 7]. Moreover, MPS calculated as the fractional synthetic rate (FSR) - a method that measures metabolism only in muscle tissue-is highly correlated with MPS determined by the three-pool AV model [7] suggesting that muscle metabolism is the primary contributor to the results. Another obvious consideration is the invasive nature of sampling from an artery and vein that drains an entire limb. Whereas any artery may be sampled, an appropriate vein must be sampled, e.g., femoral vein for leg AV balance. Catheterization of an artery and a deep forearm or femoral vein obviously must be performed with great care and under appropriate clinical conditions and ethical considerations. Thus, utilization of these models is limited mostly to clinical facilities making these methods largely unavailable for studies in healthy volunteers-both athletes and other exercisers.

Other stable isotopic tracer models have been developed to assess MPB in vivo in humans when arterial catherization may not be feasible [32]. The principle behind these methods is that the appearance of unlabeled amino acids from MPB will dilute the tracer enrichment in the muscle intracellular pool, but not arterial blood pool [33]. So, the relationship of the enrichment in the muscle intracellular fluid and arterialized blood can be used to calculate fractional breakdown rate (FBR), i.e., MPB [32]. An advantage to this model is that MPS can be simultaneously determined and NBAL calculated. FBR can be determined simultaneously with FSR by infusing two isotopes and sampling muscle tissue and arterial or arterialized venous blood [32, 34, 35]. Whereas arterial catherization is not necessary, two or more muscle biopsies are required. More recently, a pulse-bolus version for determination of FBR was developed in the Wolfe laboratory [36]. This method requires fewer biopsies and does not require an infusion of amino acids. Physiological steady state is a crucial component of these models to determine FBR. Without this steady state, such as occurs with ingestion of a source of amino acids, the relationship between MPB and amino acid transport is variable [37] and the model breaks down leading to unreliable results. Thus, it is not possible to determine MPB following exercise with ingestion of protein or amino acids using these models. Recently, a technique was developed to address this shortcoming [37], but to date it has never been validated in humans. So, to our knowledge, it is possible to measure MPB in response to an infusion or constant ingestion of steady doses of protein or amino acids with AV balance methods. However, assessing the response of MPB to a bolus ingestion of a source of amino acids remains problematic. These limitations have led to the dearth of available data on MPB in humans.

Both AV balance and FBR methods to assess MPB are limited to degradation rates of mixed muscle proteins, i.e., all proteins in the muscle. There is no resolution of breakdown on the individual protein level or even protein subfraction, e.g., myofibrillar versus mitochondrial proteins. Measuring rates of synthesis of protein subfractions has become quite common over the past decade [2, 5, 38, 39]. However, measuring breakdown rates of these protein subfractions is difficult. One approach that attempts to address this limitation is to measure 3-methylhistidine $(3 \mathrm{MH})$, which is a post-translationally methylated histidine found in myofibrillar proteins. $3 \mathrm{MH}$ is used as a marker of myofibrillar MPB because it cannot be further metabolized nor can it be reutilized for MPS. Many studies have measured urinary $3 \mathrm{MH}$ as a marker of whole body myofibrillar protein breakdown. But, of course, $3 \mathrm{MH}$ is found in tissues other than skeletal muscle, e.g., cardiac and smooth muscle, so increased urinary $3 \mathrm{MH}$ does not represent only skeletal MPB. Moreover, studies measuring $3 \mathrm{MH}$ must ensure that participants consume a meat free diet. More recently, AV balance of $3 \mathrm{MH}$ has been used to determine MPB, but this method has been used sparingly and only in clinical studies [40]. Interstitial $3 \mathrm{MH}$ recently has been measured after exercise and inactivity using microdialysis [41-43]. However, in addition to the above limitations, it must be assumed that increased $3 \mathrm{MH}$ in the interstitial fluid appears as a result of increased MPB. This method has been criticized [44] and the sensitivity of the measurement seems to be insufficient to detect changes in MPB following many forms of exercise [43]. Thus, the efficacy of using this method to assess myofibrillar MPB following exercise in humans is uncertain, particularly given the intricacies of muscle microdialysis techniques.

More recently, attempts have been made to investigate breakdown of individual proteins in muscle. One method involves measurement of decay of isotopic enrichment of individual proteins $[45,46]$. However, this method has yet to be used in a study involving exercise and nutrition. Moreover, this method measures MPB only over the course of at least several days and as much as $2-3$ weeks. Thus, the rates of MPB calculated would not be comparable to acute measurements of MPS. Whereas the time frame of MPB measurement may be applicable to what can be generated with deuterated water methods for assessing MPS [47, 48], the two methods may not be used simultaneously and NBAL cannot be determined. So, the utility of 
this method for assessing breakdown of individual proteins [45] seems to be fairly limited.

Another method recently has been developed to determine breakdown of individual proteins in muscle using proteomic analysis. Breakdown rate of each protein is calculated from the measurement of the synthesis of each protein using stable isotopic tracers combined with changes in abundance of the protein. This method has been reported in fish [49] and, more recently, in humans following exercise during low carbohydrate, high fat feeding [50]. Since determination of MPB is indirect, there are limitations that must be considered. Breakdown rates of some proteins have been reported to be negative due to a number of factors [50]. Thus, whereas this method offers a way to acquire some important information about the response of MPB to exercise and nutrition, appropriate caution should be applied with interpretation of the results.

In summary, there are a number of methods that have been used to determine the dynamic response of MPB to exercise and nutrition. Most of these methods utilize stable isotopic tracer techniques to assess MPB and the various limitations of the available methods make measurement of MPB much more difficult than MPS. Thus, there is much less information on the response of MPB to exercise and nutrition available. Nevertheless, important mechanistic information may be gleaned from these studies.

\subsection{Molecular Pathways of MPB}

Information about the response of MPB in humans also may be gleaned from measurements of changes in the response of molecular signaling of MPB pathways. These measures are made from muscle samples taken at a given, individual time point. Thus, these assessments of MPB pathways in response to exercise are mainly generated through examination of ribonucleic acid (RNA) or protein levels or indices of protein signaling/activation responses (phosphorylation, autolysation, etc). The development of the polymerase chain (PCR) methodology and subsequently quantitative real-time PCR (qRT-PCR) led to an explosion of studies using these techniques to assess gene expression [51]. Whilst qRT-PCR takes a gene by gene approach, global scale technologies such as microarrays [52] and more recently RNA-sequencing (RNA-Seq) [53] also are available to quantify the transcriptional response of muscle to exercise. The sensitivity of these molecular biology methods means that great care should be taken with sample preparation to prevent contamination with exogenous RNA that can confound findings. This sensitivity also means that very small amounts of tissue are required. RNA levels can be assessed alongside other parameters (e.g., protein levels, enzyme activity) in the same tissue sample. Human exercise studies most commonly measure changes in messenger RNA (mRNA) expression of E3 ligases, e.g., MuRF1 and atrogin1, to suggest changes in MPB with various interventions [6].

A major weakness of any assessment of RNA levels is that these do not always reflect physiological changes in muscle metabolism or mass [54]. For example, Reitelseder et al. [55] reported no change in MPB rates measured with stable isotopic tracer methods, but MuRF1 expression was increased following exercise. Additionally, calpain-3 mRNA levels were reduced $24 \mathrm{~h}$ after eccentric exercise [56], whilst calpain-3 autolysis, and presumably activity level, was increased after eccentric exercise at the same time point [57]. Thus, increased mRNA expression does not always point to increased activity of a pathway. Furthermore, the quantification of RNA levels by qRT-PCR is usually relative and so the absolute level of RNA species examined is usually unknown. Finally, the response of mRNA expression of multiple proteins may be variable $[55,58-60]$ making interpretation of these results problematic. These variable responses may suggest different functional properties of the proteins. Nonetheless, these limitations must be carefully considered when results using these methods are appraised.

When using qRT-PCR, levels of the target RNA are usually compared to a normalizer RNA, which should not change across conditions. Other normalization methods also are available [61]. The unchanging nature of this normalizer RNA often is not examined explicitly and the choice of reference gene to use as a normalizer is often accepted without critical evaluation [62]. Studies have examined suitable panels of reference genes to use for qRT-PCR in skeletal muscle [63] and tools have been developed to help researchers select suitable normalizer genes for a qRT-PCR experiment [64]. Partly in response to low reproducibility rates, guidelines for adequate reporting of qRT-PCR studies were published [65]. Along with several other excellent recommendations these guidelines include the explicit checking of normalizer RNA expression stability and level. Indeed, it is probably optimal to use more than one normalizer RNA and appropriate normalization methods [66, 67]. Nonetheless for interpretation of studies of MPB or atrophy that use qRT-PCR the reader should be aware that uncritical use of 'stock' normalizing RNA species is still widespread. Studies examining the expression of 'usual' normalizer RNAs for qRT-PCR are rare but Sunderland et al. [68] reported that the expression of several usual normalizer RNAs can be influenced by both subject age and time after exercise.

Microarrays [52] and RNA-Seq [53] both give a global overview of transcription and this information can be used to examine enriched pathways and processes or to identify 
potential markers for high or low responders. The advantage of these technologies is the broad coverage of transcriptional activity for the RNA species of interest. Microarrays and RNA-Seq also can be adapted to give information on the epigenetic state of DNA (i.e., methylation, acetylation, etc.). Microarray and RNA-Seq are both very sensitive to contamination and as with qRT-PCR, care must be taken in sample preparation. However, whilst functional events at the protein level cannot be directly inferred, the global nature of the profiling does mean that the biological context of the tissue can be inferred. The global methods can return large numbers (possibly thousands) of genes or other entities that vary with the condition of interest and making sense of these lists is challenging. The most widely adopted approach is one of enrichment or category analysis [69]. Enrichment analysis takes a list of identified genes and uses statistical testing to ask if any pre-curated biological process, function or pathway is enriched in that list and, if so, in which direction the genes change with the condition. The prototypical example of this technique is gene set enrichment analysis (GSEA) [70]. Various curated repositories provide information on whether genes belong to biological processes or pathways [71-73]. One caveat with enrichment analysis is that the information in these resources is constantly changing as new findings come to light.

\section{Response of MPB to Exercise and Nutrition}

\subsection{Exercise}

Exercise is a powerful mediator of MPB. Generally, it is thought that resistance exercise increases MPB [6]. In the first study to assess MPB using dynamic, stable isotopic tracer methods we demonstrated that mixed protein MPB was increased following resistance exercise in untrained volunteers [7]. The increase in MPB was less than the increase in MPS, so NBAL was increased. However, NBAL did not reach net positive balance during these measurements in the fasted state [1]. These MPB results generated using AV balance methodology were replicated subsequently using another stable isotopic method. The FBR of mixed muscle proteins was increased following resistance exercise in untrained individuals, but less than FSR leading to improved, but still negative NBAL [34]. Interestingly, FBR was increased for $24 \mathrm{~h}$ following exercise whereas FSR remained elevated for $48 \mathrm{~h}$. Recently, FBR also was reported to be unchanged by resistance exercise $48 \mathrm{~h}$ prior during an energy deficit [74]. Thus, it seems clear that, at least with a sufficient stimulus, resistance exercise stimulates increased mixed MPB in untrained volunteers.
Broad support for the notion that MPB is increased following resistance exercise comes from studies measuring molecular markers. Studies consistently report that muscle specific ubiquitin ligase MuRF1 mRNA expression was increased in the first few hours after resistance exercise in untrained individuals [55, 60, 75-79]. However, mRNA expression of atrogin1, another E3-ligase, reportedly decreased $[60,75,80]$ or remained unchanged $[75,81]$ following resistance exercise. Recently, Hector et al. [74] reported that a number of molecular markers of MPB were unchanged $48 \mathrm{~h}$ following resistance exercise during energy deficit conditions. These divergent responses suggest the roles of these ligases may vary. Alternately, the response of the two ligases may be dependent on fiber type $[12,77]$. It is important to note that these measures come from only a single time point, so they represent a 'snapshot' of the response. Moreover, increases in mRNA do not always lead to increased protein levels, not to mention physiological activity. Changes in mRNA expression are often not associated with dynamic measures of MPB [55]. Thus, given that the preponderance of the available data shows that expression of at least some components of the ubiquitin-proteasome pathway increase, overall these results are consistent with the dynamic measurements indicating that MPB increases in response to resistance exercise.

Training status seems to impact the response of MPB to resistance exercise. Using a cross-sectional comparison, we demonstrated that mixed muscle FBR was increased following resistance exercise in untrained individuals [35]. However, the same exercise bout (i.e., same relative exercise intensity) resulted in little, if any, increase in FBR in resistance-trained individuals. Moreover, there was no difference in resting FBR between trained and untrained individuals [35]. Subsequently, FBR was measured using a longitudinal study design before and after 8 weeks of training [82]. Resting FBR was greater following than before training. Moreover, resistance exercise increased FBR prior to training, but not after training. It should be noted that FBR was measured following exercise at the same absolute exercise intensity and during constant feeding in this study [82]. So, it is difficult to compare these results directly to the previous results [35]. On the other hand, taken together these results from different studies under varying physiological conditions support the notion that training reduces the response of MPB to resistance exercise. Stefanetti et al. [76] showed reduced MuRF1 expression with resistance exercise following 10 weeks of resistance training. This response contradicts that demonstrated in untrained individuals [55, 60, 75-79]. It is generally assumed that the response of global MPB to resistance exercise reflects the degradation of myofibrillar proteins. 
There have been attempts to refine the measurement of MPB to the breakdown of the myofibrillar protein fraction. Since $3 \mathrm{MH}$ is found only in myofibrillar proteins, measurement of $3 \mathrm{MH}$ in the muscle interstitial fluid using microdialysis techniques has been used to assess myofibrillar protein degradation. These studies report no change in interstitial 3MH following resistance exercise [41, 43]. Similarly, intense endurance exercise did not result in increased interstitial 3MH [42]. These results [42, 43, 83] may be interpreted to suggest that myofibrillar breakdown is not a major contributor to the increase in mixed MPB due to intense exercise [7, 34, 35]. However, one study demonstrated that interstitial $3 \mathrm{MH}$ increased in response to electrical stimulation, but not intense eccentric contractions [43], similar to that previously shown to increase mixed MPB [7, 34, 35]. This discrepancy suggests that measurement of interstitial $3 \mathrm{MH}$ likely is not sensitive enough to detect changes in myofibrillar protein breakdown following resistance exercise [43]. Moreover, the use and validity of this methodology has been criticized and the results questioned [44]. Thus, whereas it is intuitively satisfying to believe that degradation of myofibrillar proteins provides a major proportion of overall MPB following exercise, the precise contribution of this protein fraction to overall MPB after exercise remains to be fully elucidated.

There is even less known about the dynamic response of MPB to endurance exercise compared to resistance exercise. Early reports of increased $3 \mathrm{MH}$ excretion suggest that myofibrillar MPB is increased by endurance exercise [84]. More recently, AV balance measurements showed that MPB was increased at $10 \mathrm{~min}$, but not 60 or $180 \mathrm{~min}$, following 45 min of walking on a treadmill [85]. A recent study showed no change in FBR following $45 \mathrm{~min}$ of running at $65 \% V_{2} \mathrm{O}_{2 \text { peak }}$ [86], but the determination of MPB may have been confounded by the fact that it was measured in the vastus lateralis muscle in trained volunteers. Molecular indicators of MPB have been reported to increase in response to endurance exercise $[59,76,80,87-89]$. Thus, the consensus seems to be that resistance exercise stimulates an increase in MPB, but it is not clear what the response is following endurance exercise. Clearly, more studies need to focus on the response of MPB, particularly the dynamic physiological response, to exercise of various types.

\subsection{Combination of Nutrition and Exercise}

The role of MPB in the response of NBAL following resistance exercise and nutrition is somewhat controversial $[2,90]$. Whereas the response of MPS to protein nutrition and exercise has been studied extensively [2-5], there are methodological difficulties that make measuring the response of MPB to exercise and nutrition problematic.
The available information comes primarily from AV balance studies. Biolo et al. [8] infused amino acids systemically following a resistance exercise bout and used the three-pool AV balance model to assess muscle protein metabolism. MPS was increased during hyperaminoacidemia following exercise, but there was no increase in MPB compared to resting, fasted levels. Similarly, the combined ingestion of essential amino acids and carbohydrate prevented exercise-induced MPB [91]. Unfortunately, the available, albeit limited, molecular data do not shed much light on these responses. Branched-chain amino acids (BCAA) [9, 92], intact protein [55, 81] and essential amino acids [91] seem to have no impact on MuRF1 expression. However, there is one report of reduced atrogin1 expression with post exercise BCAA ingestion [75]. It may be that the response of UPP expression is influenced by the dose of protein ingestion. Areta et al. [58] reported increased MuRF1 expression following exercise with ingestion of 10 and $20 \mathrm{~g}$ of whey protein. However, ingestion of $40 \mathrm{~g}$ prevented the increase in mRNA levels. Unfortunately, it is unclear how these changes in mRNA levels relate to changes in MPB rates [54]. Nevertheless, it seems that hyperaminoacidemia, possibly mediated primarily by BCAA, inhibits the increase in MPB following exercise.

As with hyperaminoacidemia, hyperinsulinemia inhibits the increase in MPB following resistance exercise [91, 93]. However, no increase in MPS has been reported in response to hyperinsulinemia following exercise [91, 94, 95]. Thus, improved NBAL with carbohydrate ingestion following resistance exercise stems almost entirely from inhibited MPB. However, it should be noted that no determination of the proteins involved has ever been made. It is clear that increased synthesis of myofibrillar proteins results from resistance exercise, alone and with ingestion of amino acids [39]. Nevertheless, there is no evidence that myofibrillar protein breakdown is increased with resistance exercise [41, 43] or that hyperinsulinemia impacts any particular protein or protein fraction [96]. Thus, the changes in synthesis and breakdown due to exercise in combination with hyperinsulinemia and hyperaminoacidemia may impact completely different proteins. Consequently, the mathematical calculation of NBAL may not offer much important information. At this point, there is no way to determine the physiological relevance of this calculation in terms of the response to insulin and amino acids.

The response of MPB to exercise also has been investigated during periods of reduced energy intake resulting in an energy deficit. A $20 \%$ energy deficit in healthy, physically active young males and females resulted in an $\sim 60 \%$ decrease in MPB assessed by FBR [86]. Most molecular markers, e.g., mean chymotrypsin-like activity, 


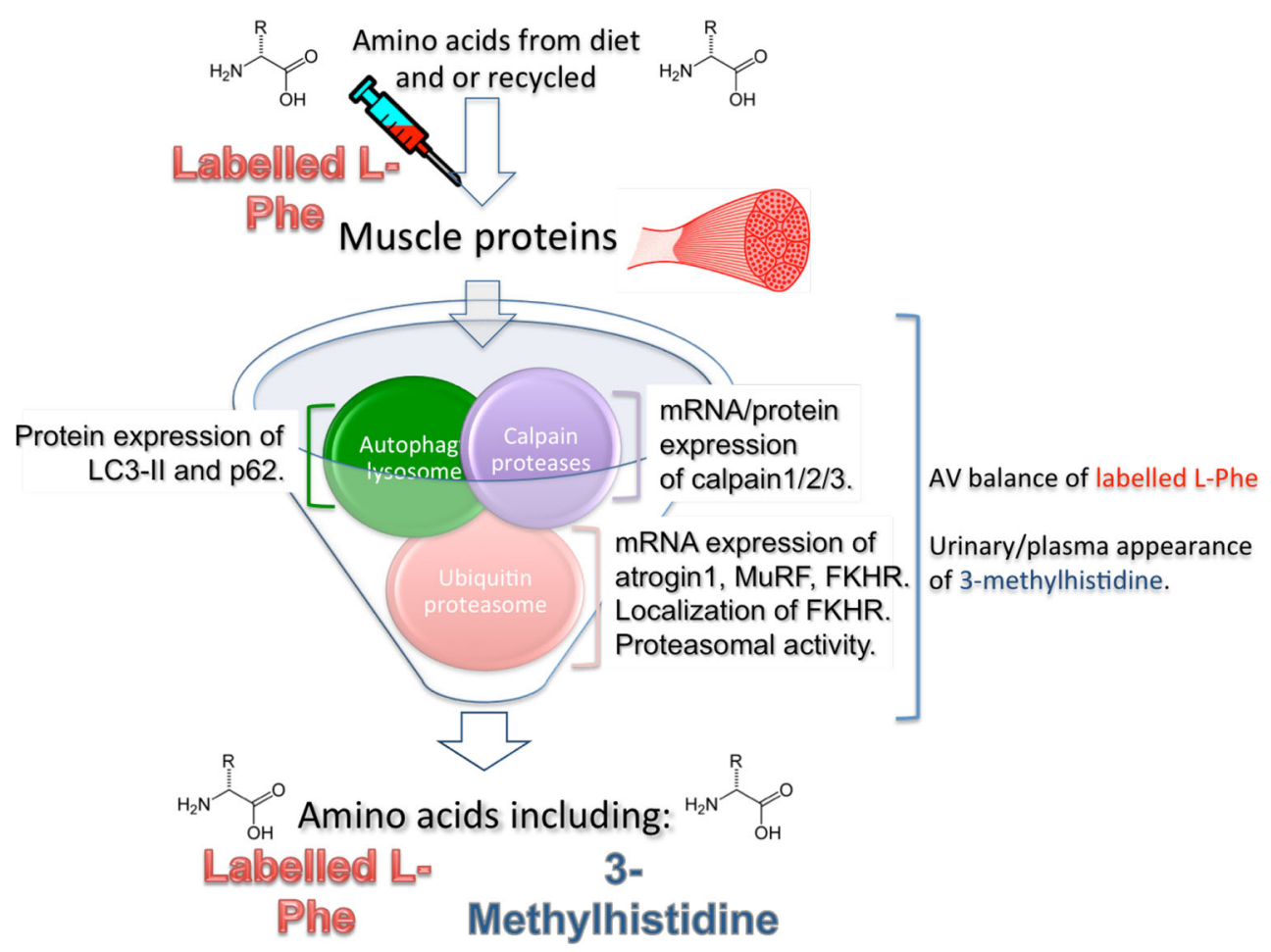

Fig. 1 Methods of assessing skeletal muscle protein breakdown (MPB). Skeletal muscle proteins are broken down by a combination of the three main protein breakdown systems. These breakdown systems do not work in isolation but rather work together to remodel skeletal muscle. (1) The calpain proteases disassemble myofibrils into smaller component parts, (2) the ubiquitin-proteasome system degrades these component into individual amino acids, and can label proteins (membrane receptors, channels and transporters) for destruction by the third system, (3) the autophagy-lysosome system, which predominantly breaks down membrane based proteins. Dynamic MPB measures use labelled amino-acid tracers (such as phenylalanine stable-isotopes) and provide a dynamic view of whole MPB.

expression of atrogin-1, of MPB were unaltered by energy deficit, but caspase- 3 activity was $\sim 11 \%$ greater than during energy balance. Alternatively, Hector et al. [74] reported that $40 \%$ energy deficit did not alter MPB (FBR) in young, overweight males. Further, no change in molecular markers of MPB was reported. The reason for the differences in these results is not certain, but may be related to the participant characteristics [74]. Nevertheless, there was no response of MPB to exercise, $45 \mathrm{~min}$ of running [86], or $48 \mathrm{~h}$ after resistance exercise [74], during energy deficit in either study. As with other nutrition and exercise situations, the paucity of studies on this topic limit a firm conclusion about the role of MPB during energy deficit at this juncture.

This response of MPB to nutrition and exercise may be explained by the physiological relationship of MPS and MPB. Resistance exercise increases MPS [7, 34, 35], likely mediated by the mammalian target of rapamycin 1 (mTORC1) signaling pathway [38, 97]. Thus, there is
3-Methylhistidine is a unique metabolite of myofibrillar protein breakdown and its appearance in blood and urine can be assumed to have come from the processes of myofibrillar protein breakdown. Skeletal muscle is the body's largest depot of myofibrillar protein so changes in plasma/urinary/interstitial 3-methylhistidine are believed reflective of skeletal MPB. Other static markers of protein breakdown include the assessment of the messenger RNA (mRNA)/protein expression/activity/localization of components of the breakdown machinery. Markers are available to estimate changes in the activity of each of the three breakdown systems. L-Phe 1-phenylalanine, AV arterio-venous, MuRF muscle ring finger protein, FKHR forkhead transcription factor

increased demand for intracellular free amino acids to supply substrate for the increased rate of MPS. Without an exogenous source of amino acids, amino acid availability for MPS is limited and MPB is increased to supply the amino acids [3]. The fact that MPS and MPB are highly correlated when measured following exercise in the postabsorptive state $[3,7,34,35]$ supports this notion. However, when amino acid availability is increased by a source of exogenous amino acids, there is no need for MPB to increase to supply amino acids for increased MPS $[3,7,34,35]$.

\section{Future Directions}

It seems clear that our understanding of the response of MPB to exercise and nutrition is incomplete. There are promising new techniques to assess the dynamic response of MPB [37, 45] that need to be validated in various 
physiological situations, including post exercise with nutrient ingestion. Simultaneous measurements of MPB rates using dynamic, stable isotopic tracer methods and static markers of MPB pathways may provide important mechanistic data to enhance our understanding. However, it must be stressed that the individual components of the machinery responsible for driving MPB do not work in isolation. Rather, each component is linked and changes in one component in isolation may or may not be responsible for driving a change in overall MPB as assessed by dynamic measures (Fig. 1). That said, the additional information that may be gleaned from studies combining these techniques in an integrated manner may add a great deal to our understanding of the contribution of the various components of the MPB machinery to changes in muscle mass, as well as muscle remodeling and adaptations to training.

\section{Conclusions}

MPB is a critical aspect of the response of muscle metabolism to an exercise bout, as well as adaptations to training. Changes in the amount of any particular protein ultimately result from the balance between the rate of synthesis and breakdown of that protein over any given time. We know that nutrition can suppress MPB following exercise [8, 91, 93]. As such, recommendations for nutritional interventions that inhibit MPB often are made. It is assumed that suppression of MPB following resistance exercise will contribute to increased NBAL and thus increased muscle mass [3, 4]. That assumption would be true if all of the inhibition was of intact, undamaged myofibrillar proteins. However, at least some of the measured global MPB resulting from exercise likely represents degradation of damaged proteins and/or proteins with rapid turnover. Degradation of these proteins likely is an important part of the adaptive process for remodeling and reconditioning muscle proteins. Thus, nutrition interventions resulting in inhibition of degradation of unnecessary or damaged proteins may actually impair adaptation to exercise training.

We simply do not know enough about the response of various individual proteins to exercise of various types. Moreover, we know next to nothing about how various nutrition interventions impact the degradation of particular proteins. Therefore, it may be a mistake to attempt to limit MPB with nutritional interventions following exercise. Finally, the changes in MPS are much greater than in MPB following exercise [5, 8, 34]. Taken together, at least until we accumulate more information on the role of degradation of various proteins in muscle remodeling, nutrition recommendations to enhance training adaptations most likely should focus primarily on the response of MPS.

Nevertheless, information on the response of MPB to exercise and nutrition provides critical information toward our understanding of muscle metabolism and exercise, as well as the influence of exercise variables and nutrition on training adaptations. This information may be useful, not only to athletes and other exercisers, but also overall metabolic health and mortality. Unfortunately, at least in humans in vivo, the technical difficulties of measuring MPB limit our current understanding of these processes. New methods for assessing MPB in various situations, including for example bolus ingestion of proteins following exercise, will be critical for evaluating the importance of changes in MPB, as well as the precise contributions of these mechanisms to muscle metabolism.

Acknowledgements This article was published in a supplement supported by the Gatorade Sports Science Institute (GSSI). The supplement was guest edited by Lawrence L. Spriet who attended a meeting of the GSSI expert panel in October 2016 and received honoraria from the GSSI for his participation in the meeting and the writing of a manuscript. He received no honoraria for guest editing the supplement. Dr. Spriet selected peer reviewers for each paper and managed the process, except for his own paper. Kevin Tipton also attended the meeting of the GSSI expert panel in October 2016 and received an honorarium from the GSSI, a division of PepsiCo, Inc. for his meeting participation and the writing of this manuscript. The views expressed in this manuscript are those of the author and do not necessarily reflect the position or policy of PepsiCo, Inc.

Open Access This article is distributed under the terms of the Creative Commons Attribution 4.0 International License (http:// creativecommons.org/licenses/by/4.0/), which permits unrestricted use, distribution, and reproduction in any medium, provided you give appropriate credit to the original author(s) and the source, provide a link to the Creative Commons license, and indicate if changes were made.

\section{References}

1. Wolfe RR. The underappreciated role of muscle in health and disease. Am J Clin Nutr. 2006;84:475-82.

2. Witard OC, Wardle SL, Macnaughton LS, et al. Protein considerations for optimising skeletal muscle mass in healthy young and older adults. Nutrients. 2016;8:181.

3. Tipton KD, Wolfe RR. Exercise-induced changes in protein metabolism. Acta Physiol Scand. 1998;162:377-87.

4. Tipton KD, Wolfe RR. Protein and amino acids for athletes. J Sports Sci. 2004;22:65-79.

5. Morton RW, McGlory C, Phillips SM. Nutritional interventions to augment resistance training-induced skeletal muscle hypertrophy. Front Physiol. 2015;6:245.

6. Pasiakos SM, Carbone JW. Assessment of skeletal muscle proteolysis and the regulatory response to nutrition and exercise. IUBMB Life. 2014;66:478-84.

7. Biolo G, Maggi SP, Williams BD, et al. Increased rates of muscle protein-turnover and amino-acid-transport after resistance exercise in humans. Am J Physiol. 1995;268:E514-20. 
8. Biolo G, Tipton KD, Klein S, et al. An abundant supply of amino acids enhances the metabolic effect of exercise on muscle protein. Am J Physiol. 1997;273:E122-9.

9. Murton A, Constantin D, Greenhaff P. The involvement of the ubiquitin proteasome system in human skeletal muscle remodelling and atrophy. Biochim Biophys Acta. 2008;1782:730-43.

10. Tokgoz Z, Bohnsack RN, Haas AL. Pleiotropic effects of ATP. $\mathrm{Mg}^{2+}$ binding in the catalytic cycle of ubiquitin-activating enzyme. J Biol Chem. 2006;281:14729-37.

11. Lee I, Schindelin H. Structural insights into E1-catalyzed ubiquitin activation and transfer to conjugating enzymes. Cell. 2008;134:268-78.

12. Kravtsova-Ivantsiv Y, Ciechanover A. Non-canonical ubiquitinbased signals for proteasomal degradation. J Cell Sci. 2012;125:539-48.

13. Bodine SC, Latres E, Baumhueter S, et al. Identification of ubiquitin ligases required for skeletal muscle atrophy. Science. 2001;294:1704-8.

14. Lecker SH, Jagoe RT, Gilbert A, et al. Multiple types of skeletal muscle atrophy involve a common program of changes in gene expression. FASEB J. 2004;18:39-51.

15. Solomon V, Goldberg AL. Importance of the ATP-ubiquitinproteasome pathway in the degradation of soluble and myofibrillar proteins in rabbit muscle extracts. $\mathrm{J}$ Biol Chem. 1996;271:26690-7.

16. Du J, Wang X, Miereles C, et al. Activation of caspase- 3 is an initial step triggering accelerated muscle proteolysis in catabolic conditions. J Clin Invest. 2004;113:115-23.

17. Jackman RW, Kandarian SC. The molecular basis of skeletal muscle atrophy. Am J Physiol. 2004;287:C834-43.

18. Tanida I, Waguri S. Measurement of autophagy in cells and tissues. Methods Mol Biol. 2010;648:193-214.

19. Mayer RJ. The meteoric rise of regulated intracellular proteolysis. Nat Rev Mol Cell Biol. 2000;1:145-8.

20. Sorimachi H, Imajoh-Ohmi S, Emori Y, et al. Molecular cloning of a novel mammalian calcium-dependent protease distinct from both $\mathrm{m}$ - and mu-types. Specific expression of the mRNA in skeletal muscle. J Biol Chem. 1989;264:20106-11.

21. Murphy RM, Verburg E, Lamb GD. $\mathrm{Ca}^{2+}$ activation of diffusible and bound pools of mu-calpain in rat skeletal muscle. J Physiol. 2006;576:595-612.

22. Dargelos E, Poussard S, Brule C, et al. Calcium-dependent proteolytic system and muscle dysfunctions: a possible role of calpains in sarcopenia. Biochimie. 2008;90:359-68.

23. Murphy RM, Lamb GD. Endogenous calpain-3 activation is primarily governed by small increases in resting cytoplasmic $\left[\mathrm{Ca}^{2+}\right]$ and is not dependent on stretch. J Biol Chem. 2009;284:7811-9.

24. Saenz A, Leturcq F, Cobo AM, et al. LGMD2A: genotype-phenotype correlations based on a large mutational survey on the calpain 3 gene. Brain. 2005;128:732-42.

25. Wolfe RR, Chinkes DL, Wolfe RR. Isotope tracers in metabolic research: principles and practice of kinetic analysis. 2nd ed. Hoboken: Wiley-Liss; 2005.

26. Thompson GN, Pacy PJ, Merritt H, et al. Rapid measurement of whole body and forearm protein turnover using a [2H5]phenylalanine model. Am J Physiol. 1989;256:E631-9.

27. Thompson GN, Pacy PJ, Ford GC, et al. Practical considerations in the use of stable isotope labelled compounds as tracers in clinical studies. Biomed Environ Mass Spectrom. 1989;18:321-7.

28. Biolo G, Chinkes D, Zhang XJ, et al. A new model to determine in vivo the relationship between amino acid transmembrane transport and protein kinetics in muscle. J Parenter Enteral Nutr. 1992;16:305-15.
29. Biolo G, Gastaldelli A, Zhang XJ, et al. Protein synthesis and breakdown in skin and muscle: a leg model of amino acid kinetics. Am J Physiol. 1994;267:E467-74.

30. Katsanos CS, Chinkes DL, Sheffield-Moore M, et al. Method for the determination of the arteriovenous muscle protein balance during non-steady state blood and muscle amino acid concentrations. Am J Physiol. 2005;289:E1064-70.

31. Tipton KD, Rasmussen BB, Miller SL, et al. Timing of amino acid-carbohydrate ingestion alters anabolic response of muscle to resistance exercise. Am J Physiol. 2001;281:E197-206.

32. Zhang XJ, Chinkes DL, Sakurai Y, et al. An isotopic method for measurement of muscle protein fractional breakdown rate in vivo. Am J Physiol. 1996;270:E759-67.

33. Chinkes DL. Methods for measuring tissue protein breakdown rate in vivo. Curr Opin Clin Nutr Metab Care. 2005;8:534-7.

34. Phillips SM, Tipton KD, Aarsland A, et al. Mixed muscle protein synthesis and breakdown after resistance exercise in humans. Am J Physiol. 1997;273:E99-107.

35. Phillips SM, Tipton KD, Ferrando AA, et al. Resistance training reduces the acute exercise-induced increase in muscle protein turnover. Am J Physiol. 1999;276:E118-24.

36. Zhang XJ, Chinkes DL, Wolfe RR. Measurement of muscle protein fractional synthesis and breakdown rates from a pulse tracer injection. Am J Physiol. 2002;283:E753-64.

37. Tuvdendorj D, Chinkes DL, Herndon DN, et al. A novel stable isotope tracer method to measure muscle protein fractional breakdown rate during a physiological non-steady state condition. Am J Physiol. 2013;304:E623-30.

38. McGlory C, Devries MC, Phillips SM. Skeletal muscle and resistance exercise training; the role of protein synthesis in recovery and remodelling. J Appl Physiol. 2016;122:541-8.

39. Witard OC, Jackman SR, Breen L, et al. Myofibrillar muscle protein synthesis rates subsequent to a meal in response to increasing doses of whey protein at rest and after resistance exercise. Am J Clin Nutr. 2014;99:86-95.

40. Vesali RF, Klaude M, Thunblad L, et al. Contractile protein breakdown in human leg skeletal muscle as estimated by [2H3]3-methylhistidine: a new method. Metabolism. 2004;53:1076-80.

41. Trappe T, Williams R, Carrithers J, et al. Influence of age and resistance exercise on human skeletal muscle proteolysis: a microdialysis approach. J Physiol. 2004;554:803-13.

42. Haus JM, Miller BF, Carroll CC, et al. The effect of strenuous aerobic exercise on skeletal muscle myofibrillar proteolysis in humans. Scand J Med Sci Sports. 2007;17:260-6.

43. Hansen M, Trappe T, Crameri RM, et al. Myofibrillar proteolysis in response to voluntary or electrically stimulated muscle contractions in humans. Scand J Med Sci Sports. 2009;19:75-82.

44. Rennie MJ, Phillips S, Smith K. Reliability of results and interpretation of measures of 3-methylhistidine in muscle interstitium as marker of muscle proteolysis. J Appl Physiol. 2008;105:1380-1 (author reply 2-3).

45. Holm L, O'Rourke B, Ebenstein D, et al. Determination of steady state protein breakdown rate in vivo by the disappearance of protein-bound tracer-labeled amino acids: a method applicable in humans. Am J Physiol. 2013;304:E895-907.

46. Holm L, Kjaer M. Measuring protein breakdown rate in individual proteins in vivo. Curr Opin Clin Nutr Metab Care. 2010;13:526-31.

47. Wilkinson DJ, Atherton PJ, Phillips BE, et al. Application of deuterium oxide $\left(\mathrm{D}_{2} \mathrm{O}\right)$ to metabolic research: just $\mathrm{D}_{2} \mathrm{O}$ it? Depends just how you $\mathrm{D}_{2} \mathrm{O}$ it! Am J Physiol. 2015;308:E847.

48. Wilkinson DJ, Cegielski J, Phillips BE, et al. Internal comparison between deuterium oxide $\left(\mathrm{D}_{2} \mathrm{O}\right)$ and $\mathrm{L}$-[ ring-13C6] phenylalanine for acute measurement of muscle protein synthesis in humans. Physiol Rep. 2015;3:e12433. 
49. Doherty MK, Brownridge P, Owen MA, et al. A proteomics strategy for determining the synthesis and degradation rates of individual proteins in fish. J Proteom. 2012;75:4471-7.

50. Camera DM, Burniston JG, Pogson MA, et al. Dynamic proteome profiling of individual proteins in human skeletal muscle after a high-fat diet and resistance exercise. FASEB J. 2017. (E-pub ahead of print. PMID: 28855275).

51. VanGuilder HD, Vrana KE, Freeman WM. Twenty-five years of quantitative PCR for gene expression analysis. Biotechniques. 2008;44:619-26.

52. Virtanen C, Takahashi M. Muscling in on microarrays. Appl Physiol Nutr Metab. 2008;33:124-9.

53. Metzker ML. Sequencing technologies-the next generation. Nat Rev Genet. 2010;11:31-46.

54. Atherton PJ, Greenhaff PL, Phillips SM, et al. Control of skeletal muscle atrophy in response to disuse: clinical/preclinical contentions and fallacies of evidence. Am $\mathrm{J}$ Physiol. 2016;311:e594-604.

55. Reitelseder S, Agergaard J, Doessing S, et al. Positive muscle protein net balance and differential regulation of atrogene expression after resistance exercise and milk protein supplementation. Eur J Nutr. 2014;53:321-33.

56. Feasson L, Stockholm D, Freyssenet D, et al. Molecular adaptations of neuromuscular disease-associated proteins in response to eccentric exercise in human skeletal muscle. J Physiol. 2002;543:297-306.

57. Murphy RM, Goodman CA, McKenna MJ, et al. Calpain-3 is autolyzed and hence activated in human skeletal muscle $24 \mathrm{~h}$ following a single bout of eccentric exercise. J Appl Physiol. 2007;103:926-31.

58. Areta JL, Burke LM, Ross ML, et al. Timing and distribution of protein ingestion during prolonged recovery from resistance exercise alters myofibrillar protein synthesis. J Physiol. 2013;591:2319-31.

59. Coffey VG, Shield A, Canny BJ, et al. Interaction of contractile activity and training history on mRNA abundance in skeletal muscle from trained athletes. Am J Physiol. 2006;290:E849-55.

60. Nedergaard A, Vissing K, Overgaard K, et al. Expression patterns of atrogenic and ubiquitin proteasome component genes with exercise: effect of different loading patterns and repeated exercise bouts. J Appl Physiol. 2007;103:1513-22.

61. Huggett J, Dheda K, Bustin S, et al. Real-time RT-PCR normalisation; strategies and considerations. Genes Immun. 2005;6:279-84.

62. Chapman JR, Waldenstrom J. With reference to reference genes: a systematic review of endogenous controls in gene expression studies. PLoS One. 2015;10:e0141853.

63. Thomas KC, Zheng XF, Garces Suarez F, et al. Evidence based selection of commonly used RT-qPCR reference genes for the analysis of mouse skeletal muscle. PLoS One. 2014;9:e88653.

64. Hruz T, Wyss M, Docquier M, et al. RefGenes: identification of reliable and condition specific reference genes for RT-qPCR data normalization. BMC Genomics. 2011;12:156.

65. Bustin SA, Benes V, Garson JA, et al. The MIQE guidelines: minimum information for publication of quantitative real-time PCR experiments. Clin Chem. 2009;55:611-22.

66. Pfaffl MW, Tichopad A, Prgomet C, et al. Determination of stable housekeeping genes, differentially regulated target genes and sample integrity: BestKeeper-Excel-based tool using pairwise correlations. Biotechnol Lett. 2004;26:509-15.

67. Vandesompele J, De Preter K, Pattyn F, et al. Accurate normalization of real-time quantitative RT-PCR data by geometric averaging of multiple internal control genes. Genome Biol. 2002;18:3.

68. Sunderland KL, Roberts MD, Dalbo VJ, et al. Aging and sequential resistance exercise bout effects on housekeeping gene messenger RNA expression in human skeletal muscle. J Strength Cond Res. 2013;27:1-7.

69. Curtis RK, Oresic M, Vidal-Puig A. Pathways to the analysis of microarray data. Trends Biotechnol. 2005;23:429-35.

70. Subramanian A, Kuehn H, Gould J, et al. GSEA-P: a desktop application for Gene Set Enrichment Analysis. Bioinformatics. 2007;23:3251-3.

71. Ashburner M, Ball CA, Blake JA, et al. Gene ontology: tool for the unification of biology. The Gene Ontology Consortium. Nat Genet. 2000;25:25-9.

72. Croft D, O'Kelly G, Wu G, et al. Reactome: a database of reactions, pathways and biological processes. Nucleic Acids Res. 2011;39:D691-7.

73. Kanehisa M. A database for post-genome analysis. Trends Genet. 1997;13:375-6.

74. Hector AJ, McGlory C, Damas F, et al. Pronounced energy restriction with elevated protein intake results in no change in proteolysis and reductions in skeletal muscle protein synthesis that are mitigated by resistance exercise. FASEB J. 2017. (E-pub ahead of print. PMID: 28899879).

75. Borgenvik M, Apro W, Blomstrand E. Intake of branched-chain amino acids influences the levels of MAFbx mRNA and MuRF-1 total protein in resting and exercising human muscle. Am J Physiol. 2012;302:E510-21.

76. Louis E, Raue U, Yang Y, et al. Time course of proteolytic, cytokine, and myostatin gene expression after acute exercise in human skeletal muscle. J Appl Physiol. 2007;103:1744-51.

77. Yang Y, Jemiolo B, Trappe S. Proteolytic mRNA expression in response to acute resistance exercise in human single skeletal muscle fibers. J Appl Physiol. 2006;101:1442-50.

78. Stefanetti RJ, Lamon S, Rahbek SK, et al. Influence of divergent exercise contraction mode and whey protein supplementation on atrogin-1, MuRF1, and FOXO1/3A in human skeletal muscle. J Appl Physiol. 2014;116:1491-502.

79. Mascher H, Tannerstedt J, Brink-Elfegoun T, et al. Repeated resistance exercise training induces different changes in mRNA expression of MAFbx and MuRF-1 in human skeletal muscle. Am J Physiol. 2008;294:E43-51.

80. Stefanetti RJ, Lamon S, Wallace M, et al. Regulation of ubiquitin proteasome pathway molecular markers in response to endurance and resistance exercise and training. Pflugers Arch. 2015;467:1523-37.

81. Dalbo VJ, Roberts MD, Hassell S, et al. Effects of pre-exercise feeding on serum hormone concentrations and biomarkers of myostatin and ubiquitin proteasome pathway activity. Eur J Nutr. 2013;52:477-87.

82. Phillips SM, Parise G, Roy BD, et al. Resistance-training-induced adaptations in skeletal muscle protein turnover in the fed state. Can J Physiol Pharmacol. 2002;80:1045-53.

83. Trappe TA, Raue U, Tesch PA. Human soleus muscle protein synthesis following resistance exercise. Acta Physiol Scand. 2004;182:189-96.

84. Carraro F, Stuart CA, Hartl WH, et al. Effect of exercise and recovery on muscle protein synthesis in human subjects. Am J Physiol. 1990;259:E470-6.

85. Sheffield-Moore M, Yeckel CW, Volpi E, et al. Postexercise protein metabolism in older and younger men following moderate-intensity aerobic exercise. Am J Physiol. 2004;287:E513-22.

86. Carbone JW, Pasiakos SM, Vislocky LM, et al. Effects of shortterm energy deficit on muscle protein breakdown and intramuscular proteolysis in normal-weight young adults. Appl Physiol Nutr Metab. 2014;39:960-8.

87. Kim HJ, Jamart C, Deldicque L, et al. Endoplasmic reticulum stress markers and ubiquitin-proteasome pathway activity in response to a 200-km run. Med Sci Sports Exerc. 2011;43:18-25. 
88. Pasiakos SM, McClung HL, McClung JP, et al. Molecular responses to moderate endurance exercise in skeletal muscle. Int $\mathrm{J}$ Sport Nutr Exerc Metab. 2010;20:282-90.

89. Jamart C, Francaux M, Millet GY, et al. Modulation of autophagy and ubiquitin-proteasome pathways during ultra-endurance running. J Appl Physiol. 2012;112:1529-37.

90. Deutz NE, Wolfe RR. Is there a maximal anabolic response to protein intake with a meal? Clin Nutr. 2013;32:309-13.

91. Glynn EL, Fry CS, Drummond MJ, et al. Muscle protein breakdown has a minor role in the protein anabolic response to essential amino acid and carbohydrate intake following resistance exercise. Am J Physiol. 2010;299:R533-40.

92. Dickinson JM, Reidy PT, Gundermann DM, et al. The impact of post exercise essential amino acid ingestion on the ubiquitin proteasome and autophagosomal-lysosomal systems in skeletal muscle of older men. J Appl Physiol. 2017;122:620-30.
93. Biolo G, Williams BD, Fleming RY, et al. Insulin action on muscle protein kinetics and amino acid transport during recovery after resistance exercise. Diabetes. 1999;48:949-57.

94. Koopman R, Beelen M, Stellingwerff T, et al. Coingestion of carbohydrate with protein does not further augment postexercise muscle protein synthesis. Am J Physiol. 2007;293:E833-42.

95. Staples AW, Burd NA, West DW, et al. Carbohydrate does not augment exercise-induced protein accretion versus protein alone. Med Sci Sports Exerc. 2011;43:1154-61.

96. Abdulla H, Smith K, Atherton PJ, et al. Role of insulin in the regulation of human skeletal muscle protein synthesis and breakdown: a systematic review and meta-analysis. Diabetologia. 2016;59:44-55.

97. McGlory C, Phillips SM. Assessing the regulation of skeletal muscle plasticity in response to protein ingestion and resistance exercise: recent developments. Curr Opin Clin Nutr Metab Care. $2014 ; 17: 412-7$. 